\title{
Mejores prácticas y la productividad, su relación en operaciones de concreto en edificaciones hondureñas 2015
}

Guillermo A. Recarte ${ }^{1}$

\section{RESUMEN}

La Carrera de Ingeniería Civil y la MICGP siguiendo una línea de investigación establecida desarrolló un estudio con el objetivo de analizar la relación entre la implementación de las mejores prácticas administrativas, propuestas por el Instituto de la Industria de la Construcción (CII), y la productividad en operaciones de concreto observada por el equipo administrativo en proyectos de edificación en Honduras durante el período del 2014 al 2015. Para este propósito, se consultó a miembros del equipo administrativo de 15 proyectos de construcción de edificios multifamiliares, comerciales, y hospitales en San Pedro Sula y Tegucigalpa; con un costo de construcción superior a los 10 millones de lempiras. Los resultados de la encuesta, luego de ser procesados, los instrumentos mostraron evidencia de confiabilidad y validez en la prueba piloto y en la consulta, posteriormente, se aplicaron herramientas estadísticas descriptivas y prueba de correlación no paramétrica de Sperman.

La evidencia muestra que aunque en los equipos administrativos de las construcciones estudiadas existe muy poca a ninguna implementación de estas prácticas, existe una relación entre la mayor implementación de las prácticas y mejores desempeños productivos en las operaciones de concreto, resulta más evidente en los datos provenientes de proyectos del Municipio del Distrito Central, probablemente por ser proporcionados por representantes del constructor. Por lo que se concluyó que la edificación hondureña mejoraría significativamente su desempeño de impulsar la implementación de dichas prácticas.

Palabras Clave: prácticas administrativas, productividad, operaciones de concreto, construcción.

\footnotetext{
${ }^{1}$ Beneficiario de una beca básica de la DICYP. Profesor del Departamento de Ingeniería Civil, UNAH Valle de Sula (UNAH VS): guillermo.recarte@unah.edu.hn
} 


\section{ABSTRACT}

The career of Civil Engineering and MICGP following a line of established research developed a study to analyze the relationship between the implementation of best management practices, proposed by the Institute of Construction Industry (CII), and productivity in concrete operations observed by the management team in building projects in Honduras during the period from 2014 to 2015. For this purpose, the members of the administrative team of 15 multi-family construction projects, commercial buildings, and hospitals in San Pedro Sula and Tegucigalpa were consulted; at a construction cost of more than 10 million Lempiras. The data, after being processed, and the instruments test for evidence of reliability and validity, both in the pilot test and consultation, then, descriptive statistics and nonparametric Spearman correlation tests were applied. Evidence shows that although the management teams of the buildings studied there is little to no implementation of these practices, there is a relationship between the largest implementation of practices and better productive performance in concrete operations, it is most evident in data from project of Central District, likely to be provided by representatives of the builder. As it concluded that the Honduran building would significantly improve their performance to boost the implementation of such practices.

Keywords: best practices, productivity, concrete operations, construction. 


\section{INTRODUCCIÓN}

El efecto nivelador que la globalización (Fondo Monetario Internacional, 2008), ha creado una presión en la competitividad. Esto se ha evidenciado en la industria de la construcción en Estados Unidos (Business Roundtable, 1983) y en Chile (Alarcón C., 2012), obligando a la industria y la academia a estudiar la manera de actualizarse, a fin de mantenerse competitivos. En Honduras (Lara, 2014), la Cámara Hondureña de la Industria de la Construcción (CHICO), ha empezado a sentir estos efectos.

El The Construction Industry Institute o Instituto de la Industria de la Construcción( CII) (2012), ha prescrito quince prácticas, con el fin de mejorar el desempeño de los proyectos: la planificación previa al proyecto, el alineamiento, a constructibilidad, la gestión de materiales, la planificación para la puesta en marcha, la formación del equipo de trabajo, la asociación o partnering, la gestión de la calidad, la implementación de resultados de investigación del Cll o similar, las lecciones aprendidas, la evaluación comparativa o benchmarking, la gestión de cambios, la prevención de disputas y resolución de conflictos, la evaluación de riesgos del proyecto, las técnicas de cero accidentes.

Peter Drucker (1991), concibe la productividad como el nuevo reto y "la verdadera fuente de ventaja competitiva". En la industria de la construcción la medición de la productividad, de acuerdo con Oglesby, Parker, y Howell (1989) implica hacer un intento por medir la efectividad y eficiencia con la que se utilizan los recursos a disposición del proyecto para cumplir con el objetivo de terminar exitosamente la obra. Mientras que Serpell (2002), la define como una medición de la eficiencia con que los recursos son administrados para completar un producto específico, dentro de un plazo establecido y con un estándar de calidad dado.

Luego de un esfuerzo de 6 años de investigación (CII Research Team 252. Craft Productivity Research Program, 2014), en Estados Unidos y Canadá, estos muestran que existe una relación significativa entre la implementación de las mejores prácticas y la mejora de la productividad laboral. Por lo que se desarrolló el presente estudio para resolver la pregunta ¿Existe está misma relación en operaciones de concreto en proyectos de edificación en Honduras en el 2014-2015? La consulta se dirigirá al equipo administrativo, dado que Oglesby, Parker y Howell (1989), sostienen que es el equipo administrativo el responsable del suministro de los recursos necesarios para el efectivo y eficiente desarrollo de las labores. 
El estudio pretende en primer lugar un aporte práctico a la industria de la construcción, una industria $(\mathrm{BCH}, 2011 ; 2014)$ que aun estando deprimida desde el 2008, hace un aporte del $6.03 \%$ al producto interno bruto (PIB) y contrata al $6.03 \%$ de la población económicamente activa entre 2008 y 2013. Se pretende incorporar nuevo conocimiento, sobre esta industria, a la Carrera de Ingeniería Civil y la MICGP. Finalmente, se busca dar un paso en la validación de instrumentos y la implementación de técnicas de medición de las prácticas y la productividad, que puedan ser usados por miembros de la industria de la construcción para hacerla más eficiente.

Metodología

El estudio fue desarrollado desde una perspectiva cuantitativa, con un diseño no experimental, realizando un corte transversal en el tiempo, con un alcance correlaciona. Para fines del estudio se utilizó la definición del CII (2012), se entiende por mejores prácticas administrativas, todo "proceso o método que, cuando se ejecuta con eficacia, conduce a una mayor rendimiento del proyecto". De esta fuente se adaptó un cuestionario, con el fin de medir la implementación de las prácticas a partir de las percepciones del equipo administrativo, utilizado una escala Likert de 5 ítems que variaba desde que el participante se manifiesta en total en desacuerdo con la proposición que se le presenta (1) hasta el participante se manifiesta en total en acuerdo con la misma (5).

En el estudio cuando se habla de medir la productividad, se refiere a la productividad laboral adoptada por el Cll (Park, Thomas, \& Tucker, 2005), quienes definen la productividad laboral como la razón entre el número de horas de trabajo real y la cantidad de unidades instaladas. Se utilizan preguntas abiertas para poder determinar la cantidad de horas hombre invertidas en cada actividad y la cantidad de unidades producidas, ver formula 1.

Se utilizó la tipología de estructuras de concreto sugerida por Park, Thomas y Tucker (2005). Los autores clasifican las estructuras como: "losas sobre suelo, elevadas, y de pavimentación; los cimientos se categorizan de acuerdo al volumen de concreto, de menos de 4 metros cúbicos, entre 4 y 15 metros cúbicos, entre 15 y 40 metros cúbicos, y más de 40 metros cúbicos; las estructuras de concreto que incluye columnas, vigas, chimeneas, zanjas revestidos, sistemas de entrepiso, muros de contención". Con estos datos se calcula la productividad laboral de acuerdo con la siguiente ecuación.

$$
\text { Productividad Laboral }=\frac{(\text { horas de trabajo efectivas })}{(\text { cantidad instalada })}
$$


De acuerdo con trabajos previos (Shan, Goodrum, Zhai, Haas, \& Caldas, 2011), el Programa de Benchmarking y Métricas (BM\&M) del Cll recomienda normalizar las medidas de productividad de 1 a 10, evitando así hacer públicas información sensible de los participantes. Debe de recordarse que tanto en la formula anterior como la siguiente, entre menos horas se inviertan por unidad, mayor es la productividad 0 puesto de otra manera, mayor número de unidades producidas con igual o menor número de horas hombre invertidas, indican una mejor productividad, ver formula 2.

$$
\mathrm{P}_{\text {Normal }}=\left(\frac{\mathrm{P}_{\text {Real }}-\mathrm{P}_{\text {Real Min }}}{\mathrm{P}_{\text {Real Max }}-\mathrm{P}_{\text {Real Min }}}\right) \quad\left(\mathrm{P}_{\text {Norm Max }}-\mathrm{P}_{\text {Norm Min }}\right)+\mathrm{P}_{\text {Norm Min }}
$$

Dónde: PNormal es la productividad normalizada, PReal es la medición real de la productividad, PReal Min y PReal Max son las mediciones reales mínima y máxima de la productividad en la tarea. PNormal min tiene un valor de $1 \mathrm{y}$ representa la mejor evaluación de la productividad; PNormal max tiene un valor de 10 y representa la peor evaluación.

En la población se consideraron los equipos administrativos de proyectos de construcción de edificios multifamiliares, comerciales, educativos, iglesias y hospitales con un costo de construcción superior a los 10 millones de lempiras cuya construcción haya sido concluida en el período 2014-2015, o que esté en proceso y permita recabar datos para análisis de la productividad.

Se obtuvo un listado de los permisos de construcción del Municipio de San Pedro Sula (MSPS) y del Municipio del Distrito Central (MDC), identificaron, inicialmente, un total de 55 proyectos que cumplían estos criterios, 25 en el Distrito Central y 30 en San Pedro Sula. La información fue proporcionada por el Colegio de Ingenieros Civiles de Honduras $(\mathrm{ClCH})$ y a la Gerencia de Infraestructura de la Municipalidad del Distrito Central. Se obtuvo una muestra pequeña probabilística por estratos, utilizando el proceso descrito por Hernández Sampieri, et al (2014).

Se envió un cuestionario utilizando la aplicación Formularios de Google y se les dio seguimiento vía teléfono y Whatsapp. Se recibió respuesta de 15 equipos administrativos, 8 en MSPS y 7 en el MDC, lo que representa un error estándar de 0.11 . Es de observar que la muestra resultante, se vio limitada por carencias en las bases de datos de ambas municipalidades. 
Para realizar el análisis de los datos se utilizó el SPSS versión 15. Para la exploración y descripción de las variables de estudio se utilizaron herramientas de la estadística descriptiva. El análisis de la confiabilidad realizó utilizando el alfa de Cronbach, tanto en la prueba piloto como el cuestionario final. El análisis para la prueba de hipótesis, se realizó utilizando, una prueba no paramétrica, el coeficiente de correlación Spearman. Las tablas y gráficos se prepararon en Microsoft Excel.

\section{RESULTADOS}

En los primeros días del mes de diciembre se envió un cuestionario, de los 25 proyectos que cumplían los criterios de selección en la prueba piloto se recibieron 8 respuestas, pese al seguimiento dado, no se lograron más participaciones. Los instrumentos utilizados para medir las prácticas administrativas aplicadas, todos obtuvieron evaluaciones niveles aceptables, con valores de alfa entre 0.776 a 0.990 . En la etapa final, todas los conceptos de las prácticas obtuvieron niveles de confiabilidad dentro de límites aceptables con valores de alfa entre de 0.817 y 0.992 .

Los equipos que respondieron $53 \%$ estaban ubicados en MSPS y $47 \%$ en el MDC. Los participantes se describieron como miembros de la organización constructora en $73 \%$, un $7 \%$ manifestó que participaban en el proyecto bajo una estrategia de contratación de diseño y construcción, el restante $20 \%$ eran representantes de la organización propietaria del proyecto o de la supervisión. Los edificios comerciales, con $47 \%$ y los edificios multifamiliares totalizan el $46 \%$, solo participó una edificación de un hospital en el estudio. Del total de edificaciones el $40 \%$ se ejecutaban mediante una estrategia de contratación de proyectos "llave en mano", 33\% de los proyectos fueron realizados por medio de contratos de diseño y construcción, mientras que tres se realizaban utilizando la administración de construcción y solo uno la administración de proyectos.

\section{Las prácticas administrativas}

En esta sección presentamos los resultados obtenidos al medir la percepción en los equipos administrativos en cuanto a la implementación de estas prácticas. En la ilustración No.1, se aprecia las dos prácticas mejor evaluadas, planificación previa al proyecto y la constructibilidad. En la primera, las respuestas de los equipos apuntan a que se ha realizado algún trabajo en su implementación, al responder estar "de acuerdo" o "totalmente de acuerdo". El aspecto con la menor evaluación fue la utiliza- 
ción del Índice Valoración del Alcance del Proyecto (PDRI), obtuvo una evaluación teniendo aproximadamente un $40 \%$ de los participantes una opinión desfavorable.

La segunda, la constructibilidad, implica la incorporación temprana en la vida del proyecto de la experiencia constructiva. Casi todos los aspectos evaluados obtuvieron opiniones favorables, excepto cuatro, coincidentemente, los aspectos con las más bajas evaluaciones, son los que representan la presencia de herramientas que sistematizan dichas prácticas.

\section{Gráfico 1. Evaluación de las prácticas administrativas en operaciones de concre- to en edificaciones hondureñas.}

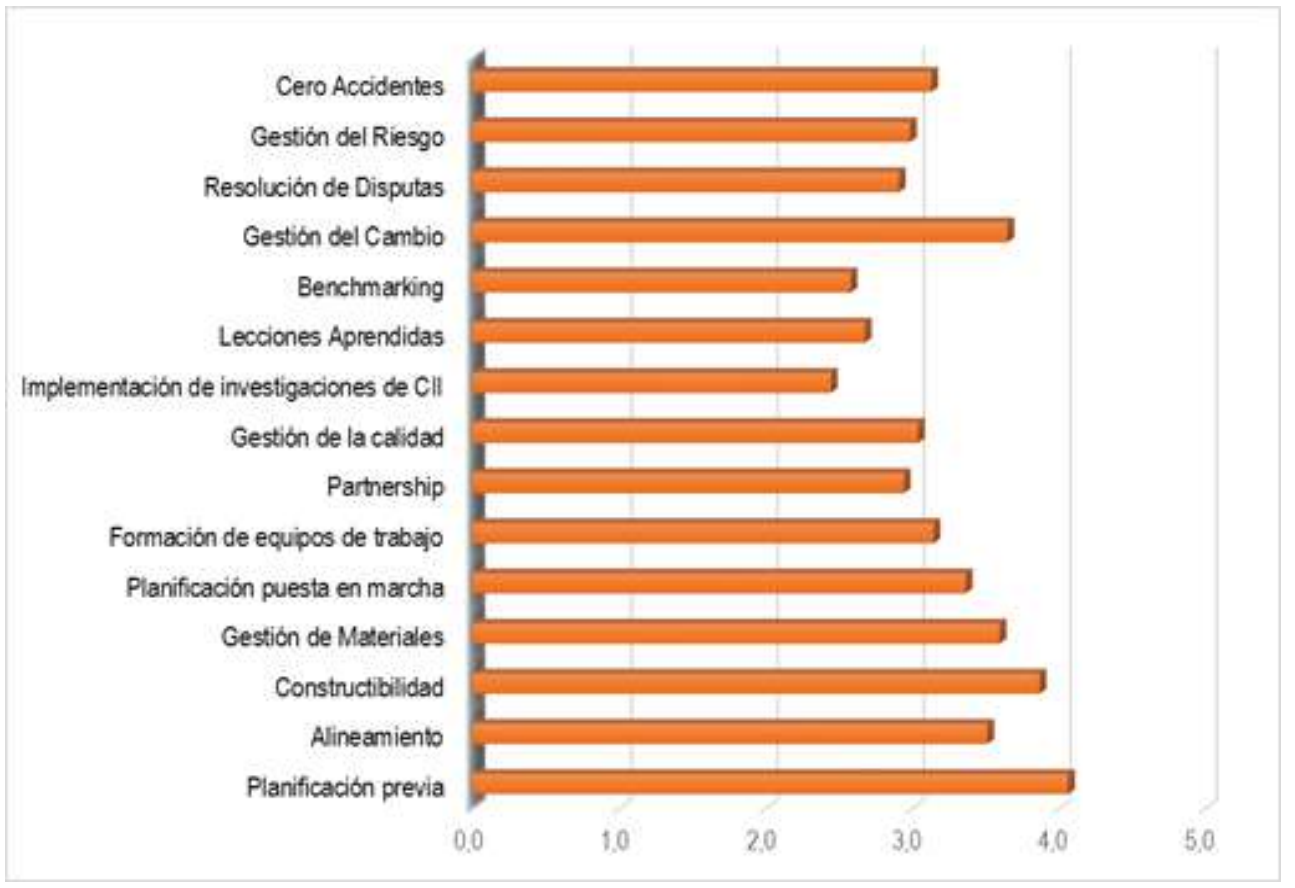

Elaboración propia en base a encuesta.

Mientras que las prácticas con las evaluaciones más bajas son: Implementación de investigaciones de CII, la de lecciones aprendidas, y la evaluación comparativa, como se observa en la Gráfico No.1. En las tres prácticas la mayor parte de los aspectos tuvieron evaluaciones intermedias, pero al igual que en la constructibilidad, son aquellos aspectos relacionados con la sistematización del proceso de mejora continua, los que tienen las evaluaciones más bajas. Las demás prácticas obtuvieron evaluaciones que implican poca implementación de estas prácticas. En general al considerar las prácticas administrativas, casi el $67 \%$ de los participantes le otorgaron una evalua- 
ción de baja a intermedia. No se encontraron diferencias significativas de cómo se perciben las prácticas administrativas, entre las diferentes ubicaciones, organización a la que pertenece el participante, uso de la edificación, estrategia de contratación, costo del contrato, avance del proyecto o tamaño de la empresa ejecutando la construcción.

\section{La productividad en operaciones de concreto en la industria de la construcción hondureña}

Con la información recopilada se procedió al cálculo de la productividad laboral haciendo uso de la ecuación No. 1 y la ecuación No. 2, para normalizar los resultados, para cada una de las categorías. Los resultados oscilan entre un rango de 1 a 10 , siendo 1 la mejor productividad y 10 la menor productividad, permitiendo organizar de mejor a menor productividad de los proyectos en sus respectivas categorías.

Las operaciones involucrando losas sobre suelo el proyecto No. 14, tuvo la mejor productividad en este tipo de operación, mientras que el proyecto No. 1 la peor del grupo observado. Al evaluar la productividad del proyecto, el proyecto 14 obtuvo el mejor resultado mientras que el proyecto No. 8 obtuvo la peor medición de productividad, los resultados se presentan en la tabla No.1.

Lo que se observó es cierta discrepancia en los resultados obtenidos de la productividad, por lo que se optó a realizar pruebas de correlación entre diferentes tipos de productividad, sorteando los grupos por ubicación, organización a la que pertenece el participante, uso de la edificación, estrategia de contratación, costo del contrato, avance del proyecto o tamaño de la empresa ejecutando la construcción. Se apreció que en los proyectos en MDC, las diferentes mediciones de la productividad, tenían relaciones más consistentes que en SPS.

\section{La relación de las mejores prácticas y la productividad en operaciones de concreto en la industria de la construcción hondureña}

Al realizar la prueba de correlación de Spearman, para la prueba de hipótesis, entre las diferentes prácticas administrativas y las mediciones de la productividad realizadas en las operaciones de concreto, inicialmente, se obtuvieron resultados confusos, la evidencia mostraba que existía relación entre algunas prácticas y la productividad en la construcción de cimientos con un volumen entre 4 a 15 metros cúbicos. La relación no está tan clara cuando se observan los resultados que da la prueba con las otras mediciones de la productividad. 
Tabla 1. Productividad obtenida por proyecto para diferentes operaciones de concreto en edificaciones hondureñas. Se omite información de los proyectos 3 y 13 por carecer de mediciones.

\begin{tabular}{|c|c|c|c|c|c|c|c|c|c|c|c|c|c|}
\hline 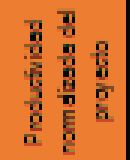 & 高 & $\begin{array}{l}8 \\
8 \\
6\end{array}$ & $\begin{array}{l}8 \\
\text { ○े } \\
\end{array}$ & $\frac{8}{8}$ & 离 & 量 & 응 & 查 & 宓 & 근 & 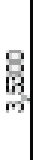 & 8 & $\frac{8}{0}$ \\
\hline 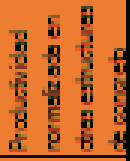 & $\begin{array}{c}8 \\
\vdots \\
\vdots \\
6 \\
\end{array}$ & $\frac{8}{\frac{8}{m}}$ & 勇 & 胥 & & 영 & $\frac{8}{8}$ & $\begin{array}{l}8 \\
8 \\
-1\end{array}$ & 恋 & 查 & 弯 & 8 & \\
\hline 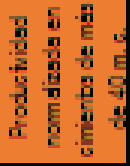 & 要 & 옹 & 8 & 궁 & & 查 & 查 & & 勇 & & & : & 8 \\
\hline 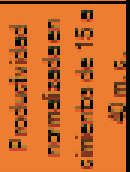 & 웅 & 惑 & 8 & 㝵 & & 查 & \begin{tabular}{l}
8 \\
8 \\
\hdashline \\
\hdashline
\end{tabular} & & 음 & & & 8 & 查 \\
\hline 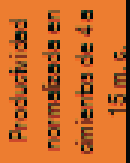 & 8 & 웋 & $\begin{array}{l}8 \\
8 \\
6 \\
6\end{array}$ & 㚆 & 영 & 煦 & 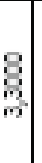 & 焉 & 空 & & & 8 & 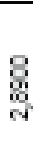 \\
\hline 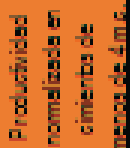 & $\begin{array}{l}8 \\
\frac{9}{9} \\
\text { m }\end{array}$ & 量 & 웅 & 各 & 离 & 㝵 & 量 & 量 & 욤 & 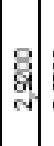 & 查 & 8 & $\$$ \\
\hline 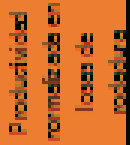 & 8 & 查 & 웅 & 查 & 8 & 穿 & 胥 & 영 & 量 & 杲 & & 8 & 8 \\
\hline 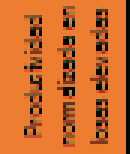 & $\begin{array}{c}8 \\
\frac{8}{5} \\
\text { m. }\end{array}$ & 量 & 㝵 & ले & 8 & 㝵 & $\begin{array}{l}8 \\
8 \\
0\end{array}$ & $\frac{8}{8}$ & 究 & 查 & 8 & 8 & 要 \\
\hline 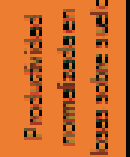 & 8 & 影 & 量 & 觮 & 离 & 恋 & 查 & 各 & 8 & 㝵 & & 审 & 量 \\
\hline$\frac{\mathrm{P}}{\frac{\mathrm{g}}{5}}$ & $\begin{array}{l}2 \\
0 \\
0 \\
\frac{9}{a}\end{array}$ & $\begin{array}{c}y \\
2 \\
0 \\
0 \\
\frac{0}{5} \\
\frac{5}{\alpha}\end{array}$ & 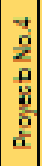 & 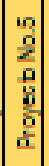 & 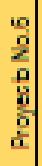 & 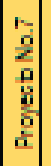 & 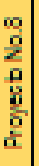 & 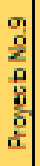 & $\begin{array}{l}\frac{9}{9} \\
\frac{1}{2} \\
\frac{9}{9} \\
\frac{5}{2}\end{array}$ & 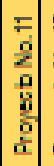 & 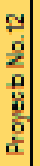 & 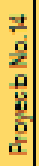 & 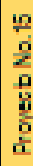 \\
\hline
\end{tabular}


Tabla 2 Relación entre las prácticas administrativas y la productividad, en el M.D.C. en diferentes operaciones de concreto en edificaciones hondureñas.
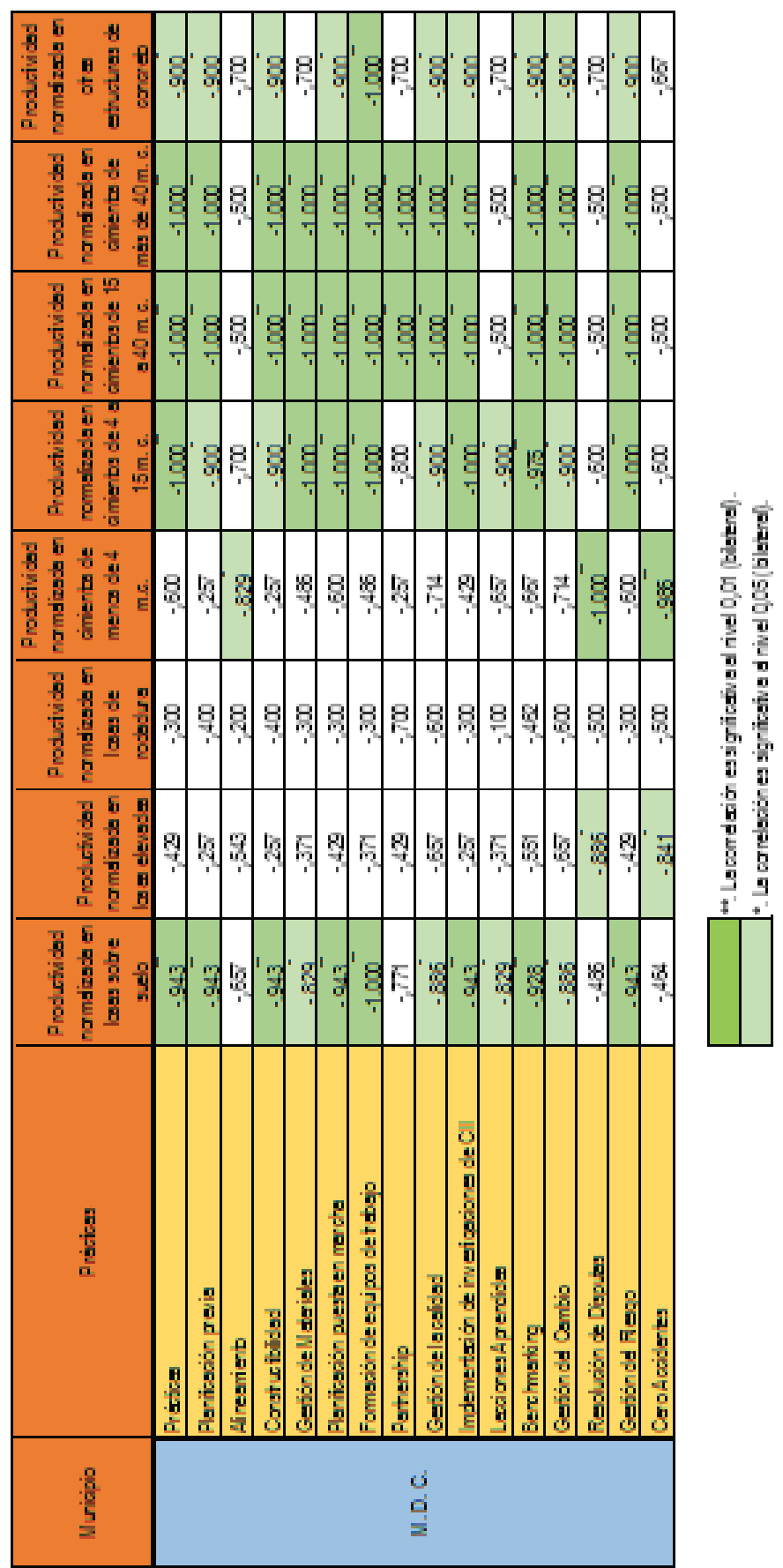
Con el objetivo de entender porque esta relación solo se presentaba en un tipo de operación, se hicieron pruebas de correlación, clasificando los datos de acuerdo a diferentes variables, se observó diferencia en cómo se manifiesta la relación en el MSPS y el MDC.

En el MDC, se obtuvo evidencia significativa que muestra claramente la existencia de esta relación. Primero, todas las prácticas, mostraron resultados estadísticamente significativos en casi todas las operaciones de construcción con excepción de las losas de rodadura, como se puede apreciar en la tabla No.2; Segundo, todas las prácticas sin excepción mostraban relaciones en la dirección de a mayor implementación de prácticas mejor productividad. Esto nos llevó a preguntarnos por qué en el MDC es más clara esta relación, la respuesta es la fuente de los datos de la productividad, se observó que 6 de los 7 participantes del MDC son miembros de la organización constructora. Se hicieron sortearon los datos de diferente maneras, para comprobar si existía otra explicación y las pruebas de correlación, no mostraban ninguna otra posible explicación.

\section{DISCUSIÓN}

Prácticas como la planificación previa del proyecto presenta las mejores evaluaciones, significando que son las que se ha hecho alguna labor de implementación al respecto. Pero la característica que obtuvo la más baja evaluación, es precisamente el PDRI (Cho \& Gibson Jr., 2001), una herramienta que ayuda a sistematizar la toma de decisiones a la organización propietaria y a analizar la definición del alcance del proyecto. La constructibilidad según Tatum, Vanegas, y Williams (1985), requiere de la identificación de posibles temas de constructibilidad de acuerdo a los objetivos del proyecto y oportunidades de mejora, así como realizar estudios especiales enfocados en temas claves. También mostró poca implementación en los aspectos que indican una sistematización de esta práctica.

Entre las prácticas hay cinco que tienen algún tiempo presente en la literatura de otras industrias, y en la industria de la construcción a nivel internacional han sido discutido desde los años ochenta, estas son la gestión de la calidad (Alarcón \& Ashley, 1996), la gestión del cambio (lbbs, Wong, \& Kwak, 2001), la prevención de disputas y resolución de conflictos (Thompson, Vorster, \& Groton, 2000), la gestión del riesgo (Walewski \& Gibson Jr., 2003) y la práctica de cero accidentes (Hinze \& Godfrey, 2003); todas tienen en común que requieren un cambio en la cultura de las 
organizaciones, tanto a nivel corporativo como a nivel de proyecto; todas recibieron evaluaciones que indican poca implementación; y todas recibieron las evaluaciones más baja en los aspectos que apuntan hacia la sistematización de decisiones y/o a los cambios culturales necesarios.

Finalmente, las restantes tres prácticas, que fueron las que obtuvieron evaluaciones aún más bajas indicando que los participantes perciben las acciones en contra de su implementación, están relacionadas con la obtención del aprendizaje organizacional necesario para cambiar los proceso internos de las organizaciones a fin de poder ser más competitivas, estas son la implementación de resultados de investigaciones del Cll o similares (Chinowsky, 2008), la utilización de programas de lecciones aprendidas (Fisher, Desphande, \& Livingston, 1998) y la evaluación comparativa o benchmarking (Lee, Thomas, \& Tucker, 2004). Lo anteriormente expuesto es coherente con la caracterización que hace Serpell (2002) sobre la industria de la construcción, reacia al cambio, aferrándose al tradicionalismo y a las experiencias pasadas.

En cuanto a los resultados obtenidos en las mediciones de la productividad, Oglesby, Parker, y Howell (1989), recomiendan diferentes medios directos para la obtención de esta medida. Este no fue el caso, se tuvo que preguntar una serie de datos a fin de poder obtener una medida de la productividad en los proyectos y no siempre quien respondió era parte del equipo encargado de la construcción, esto se evidenció al analizar la información, tal como se explicó anteriormente.

Futuras investigaciones deben de orientarse a adaptar programas de mejora a la productividad a nuestra realidad, el desarrollo e implementación de nuevas prácticas que hagan más competitiva la industria de la construcción, y un cambio cultural de la industria que promueva la innovación como fuente es competitividad.

El trabajo, como la mayor parte de los emprendimientos no se encontró libre de obstáculos que se fueron sorteando, el primero, fue la carencia de bases de datos confiables y actualizados de los proyectos de construcción. Segundo, una cultura orientada a no participar en este tipo de estudios. Y tercero, faltan iniciativas de mejora de la productividad, por lo que dificulta obtener información de fuentes primarias confiables.

En el desarrollo de este trabajo, los datos recolectados y analizados permitieron obtener una fiel descripción del grado de implementación de cada una de las prácticas estudiadas, cumpliendo el primer objetivo específico y se contestó la primera pregunta de investigación. La utilización de mediciones de la productividad normalizada, y 
haciendo uso de la tipología de Park, Thomas, y Tucker (2005), es ideal para poder llevar a cabo análisis comparativos entre diferentes proyectos, sin tener que presentar información considerada delicada por los participantes, por lo que el segundo objetivo específico, caracterizar la productividad en estos proyectos, se alcanzó y la segunda pregunta de investigación también fue contestada.

Finalmente, al analizar los datos, en especial los provenientes del MDC, la evidencia estadística soporta la hipótesis general y las hipótesis auxiliares de trabajo, ya que el índice de implementación de las prácticas también como todas las prácticas se encontraron significativamente relacionadas con casi todas las operaciones de construcción.

\section{CONCLUSIONES}

- Los equipos administrativos, en general perciben las prácticas administrativas con poca implementación en las operaciones de concreto en edificaciones hondureñas entre el 2014 y 2015.

- Se pudo obtener mediciones de la productividad, que permitieron caracterizar la misma en diferentes proyectos, sin comprometer información confidencial, en diferentes operaciones de concreto en edificaciones hondureñas entre el 2014 y 2015.

- Se obtuvo evidencia de la existencia de una relación entre la implementación de las mejores prácticas administrativas y la productividad en operaciones de concreto en proyectos de edificación en Honduras en 2014-2015, implicando que sí la industria desea mejorar su desempeño, en términos de productividad, deberá de hacer esfuerzos por implementar estas prácticas.

\section{AGRADECIMIENTOS}

A la DICYP; CRI, la Carrera de Ingeniería Civil; al CIl; y a las autoridades del MDC, $\mathrm{CICH}$ y miembros de la industria que apoyaron. 


\section{REFERENCIAS BIBLIOGRÁFICAS}

Alarcón C., L. F. (2012). Herramientas para Identificar y Reducir Pérdidas en Proyectos de Construccion. Revista Ingeniería de Construcción, 15, 37-45.

Alarcón, L. F., \& Ashley, D. B. (1996). Modeling project performance for decision making. Journal of Construction Engineering and Management, 122(3), 265-273.

BCH. (2011). Honduras en cifras. 2008-2010. Obtenido de www.bch.hn: http://www.bch.hn/download/honduras_en_cifras/hencifras2008_2010.pdf

BCH. (2014). Honduras en Cifras. 2011 - 2013. Obtenido de Banco Central de Honduras: http://www.bch.hn/download/honduras_en_cifras/hencifras2011_2013.pdf

Business Roundtable. (1983). More construction more money. Summary report of the Construction Industry Cost Effectiveness Project., Washington, D.C. Obtenido de http://faculty.ce.berkeley.edu/tommelein/BRTMoreConstructionForTheMoney.pdf

Chinowsky, P. S. (2008). Staircase Model for New Practice Implementation. Journal of Management in Engineering, 24(3), 187-195.

Cho, C.-S., \& Gibson Jr., G. E. (Diciembre de 2001). Building project scope definition using project definition rating index. Journal of Architectural Engineering, 7(4), 115-125.

CII Research Team 252. Craft Productivity Research Program. (2014). Construction Productivity Research Program. Phase V. The University of Texas at Austin, Construction Industry Institute.

Drucker, P. F. (noviembre-diciembre de 1991). The new productivity challenge. Harvard Business Review, 69(6), 2 - 8. Obtenido de https://hbr.org/1991/11/the-new-productivity-challenge

Fisher, D., Desphande, S., \& Livingston, J. (1998). Modeling the lessons learned process. The University of New Mexico, Department of Civil Engineering .

Fondo Monetario Internacional. (Mayo de 2008). Globalization: A Brief Overview. Obtenido de International Monetary Fund: http://www.imf.org/external/np/exr/ib/2008/053008.htm

Hernández Sampieri, R., Fernández Collado, C., \& Baptista Lucio, P. (2014). Metodología de la Inestigación (6ta. ed.). México D.F., México: McGraw-Hill Education.

Hinze, J. W., \& Godfrey, R. (2003). An evaluationn of safety performance measures for construction projects. Journal of Construction Research ,, 4(1), 5-15.

Ibbs, C. W., Wong, C. K., \& Kwak, Y. H. (2001). Project Change Management System. Journal of Management in Engineering Vol, 17(3), 159-165.

Lara, B. (4 de agosto de 2014). Economía: Medio millón de empleos ha perdido el sector construcción en Honduras. Diario La Prensa, pág. 56. Obtenido de http://www.laprensa.hn

Lee, S.-H., Thomas, S. R., \& Tucker, R. L. (2004). Effective practice utilization using performance prediction software. Journal of Construction Engineering and Management, 130(4), 576-585.

Oglesby, C. H., Parker, H. W., \& Howell, G. A. (1989). Productivity Improvement in Construction. New York: McGraw Hill, Inc. 
Park, H.-S., Thomas, S. R., \& Tucker, R. L. (2005). Benchmarking of Construction Productivity. Journal of Construction Engineering and Management, 772 - 778.

Serpell B., A. (2002). Administracion de operaciones de construcción (2da. ed. ed.). México: Alfaomega.

Shan, Y., Goodrum, P. M., Zhai, D., Haas, C. T., \& Caldas, C. H. (Marzo de 2011). The impact of management practices on mechanical construction productivity. Construction Management and Economics, 29(3), 305-316.

Tatum, C. B., Vanegas, J. A., \& Williams, J. M. (1985). Constructability improvement during conceptual planning. Stanford University, Department of Civil Engineering.

The Construction Industry Institute. (2012). Cll Best Practices Guide: Improving Project Performance. Implementation Resource 166-3 Version 4.0, Implementation Strategy Committee.

Thompson, R. M., Vorster, M. C., \& Groton, J. P. (2000). DInnovations to manage disputes: DRB and NEC. Journal of Management in Engineering, 16(5), 51-59.

Walewski, J., \& Gibson Jr., G. E. (2003). International project risk assessment: methods, procedures, and critical factors. The University of Texas at Austin, Construction Industry Institute Project Team 181. 